\title{
The Current and Future Status of Biomarkers in Osteoarthritis
}

In this issue of The Journal, Aslam and colleagues ${ }^{1}$ examined blood and urine biomarker levels and their association with a variety of hand osteoarthritis (OA) phenotypes. They report that serum levels of hyaluronic acid (HA) and COMP (cartilage oligomeric matrix protein) were associated with the presence of hand OA.

While among the first to study hand OA, this study is one of many reporting a positive association of these 2 serum biomarkers with the presence of OA. COMP is a constituent of hyaline articular cartilage and, with damage to cartilage, is released from cartilage into synovial fluid and hence into the circulation. Serum HA is a macromolecular glycosaminoglycan found throughout many soft tissues in the body. In arthritis, levels of HA are thought to be related to synovitis, although this relationship has been demonstrated in rheumatoid arthritis (RA) and not specifically in $\mathrm{OA}^{2}$. While some studies evaluating the relationship of these biomarkers to the presence or severity of OA have been negative, most have shown a positive association ${ }^{3,4}$.

The study by Aslam, et $a l^{1}$ constitutes one of an increasing number of articles on systemic markers of joint metabolism and their relationship with OA. The goal of this editorial is to ask what insights these studies have provided into the biology and diagnosis of OA and the likely future value of biomarker studies for the clinical care of patients with OA.

As part of a US National Institutes of Health effort to make sense of the burgeoning literature on biomarkers in $\mathrm{OA}$, Bauer et $a l^{5}$ provided a framework to categorize biomarker studies into an easily remembered BIPED classification (Burden of disease, Investigative, Prognostic, Efficacy and Diagnostic; see Table 1). Van Spil and colleagues $^{4}$, in a systematic review of biomarker literature in OA, noted that most studies focus on diagnostic markers, examining biomarker levels in patients with versus those without OA. The good news is that some markers, such as COMP and HA, have differentiated patients with and without OA. Unfortunately, that does not answer the important question: whether these biomarkers could be used as diagnostic tests to determine whether a patient has OA.
The first problem here is that, notwithstanding American College of Rheumatology classification criteria for OA, there is no widespread acceptable clinical definition of OA. Radiographic evidence of OA is a late feature of disease, and many people with chronic joint pain who have normal radiographs may well have $\mathrm{OA}^{6}$. If magnetic resonance imaging is used to look for evidence of OA, there is no accepted threshold for when disease is present. Thus, defining disease and characterizing which joints are affected is difficult.

The second problem is that, while these biomarkers separate those with clear-cut clinical OA from those without it, their diagnostic sensitivity and specificity for disease is usually poor, not attaining levels that would suggest they are good diagnostic tests. There are many inherent barriers to the development of one or more of these measures as diagnostic tests for OA. First, many of these molecules arise from soft tissues throughout the body, not just in the knee or hand joints. The majority are produced in the spine. Thus, elevated levels do not necessarily reflect disease in 1 or more peripheral joints. Second, biomarkers are generated initially within a joint and then get released into the synovial fluid, where they may or may not be cleared into the blood. Clearance rates and levels in blood or urine depend on synovial vascularity. Biomarker epitopes may be partly metabolized when they enter the bloodstream and course through organs before excretion, and degradation may affect their levels. Urinary levels are dependent on the mechanism of clearance and how much of it is cleared by the kidney. The level of a given marker is also affected by physical activity levels ${ }^{7}$ and fluctuates by time of day ${ }^{2}$. Given the multitude of factors affecting the levels of systemic biomarkers, they may never perform acceptably as diagnostic tests for OA in 1 or more peripheral joints.

So if diagnostic studies of systemic biomarkers are not likely to provide a diagnostic test, of what value are they? First, they can provide insights into the biology of the disease. For example, the elevation of serum HA in the study in this issue and in others among those with OA raises the question of where the HA originates and whether it

\section{See Biomarkers in hand OA, page 938}


Table 1. Summary of "BIPED" (Burden of disease, Investigative, Prognostic, Efficacy and Diagnostic) biomarker classification for osteoarthritis (OA). Adapted from Bauer, et al. Osteoarthritis Cart 2006;14:723-7; with permission.

\begin{tabular}{|c|c|c|c|c|c|}
\hline Definition & $\begin{array}{l}\text { Biomarker associated } \\
\text { with extent or severity } \\
\text { of OA }\end{array}$ & $\begin{array}{l}\text { Biomarker not yet } \\
\text { meeting criteria for } \\
\text { another category }\end{array}$ & $\begin{array}{l}\text { Predicts onset or } \\
\text { progression }\end{array}$ & $\begin{array}{l}\text { Indicative or predictive } \\
\text { of treatment efficacy }\end{array}$ & $\begin{array}{l}\text { Differentiates diseased } \\
\text { from non-diseased }\end{array}$ \\
\hline Subjects & Must have OA & NA & $\begin{array}{l}\text { With and/or without } \\
\text { OA }\end{array}$ & With OA & With and/or without OA \\
\hline Design & $\begin{array}{l}\text { Cross-sectional, case- } \\
\text { control }\end{array}$ & NA & Longitudinal & Controlled trial & $\begin{array}{l}\text { Cross-sectional or } \\
\text { case-control }\end{array}$ \\
\hline Analysis & $\begin{array}{l}\text { Correlation of biomarker } \\
\text { with burden; percent of } \\
\text { variance explained }\end{array}$ & NA & $\begin{array}{l}\text { Sensitivity, specificity } \\
\text { to predict poor or good } \\
\text { prognosis; if continuous; } \\
\text { consider variance } \\
\text { explained }\end{array}$ & $\begin{array}{l}\text { Risk or OR with } 95 \% \text { CI } \\
\text { among successfully vs } \\
\text { unsuccessfully treated }\end{array}$ & $\begin{array}{l}\text { Sensitivity, specificity, LR } \\
\text { AUC from ROC curve }\end{array}$ \\
\hline Criteria & $\begin{array}{l}\text { Significant association } \\
\text { between marker and } \\
\text { extent or severity of } \\
\text { OA }\end{array}$ & NA & $\begin{array}{l}\text { Significant association } \\
\text { between marker and onset } \\
\text { or progression of OA }\end{array}$ & $\begin{array}{l}\text { Significant association } \\
\text { between marker and } \\
\text { treatment effect }\end{array}$ & $\begin{array}{l}\text { Significant association } \\
\text { between marker and OA } \\
\text { diagnosis }\end{array}$ \\
\hline
\end{tabular}

LR: Likelihood ratio; AUC: area under the curve; ROC: receiver-operating characteristic curve; NA: not applicable.

reflects active synovitis in OA (as it does in RA). As is increasingly recognized, $\mathrm{OA}$ is a disease affecting all of the structures within a joint. As van Spil, et al note ${ }^{4}$, most biomarker studies in OA have focused on cartilage metabolites, and far fewer on molecules originating in synovium or bone. Although so far the studies investigating bone markers have tended to be negative, they may not sample metabolic processes that reflect the dynamic processes occurring in bone in OA. Additional such studies in which there is a more intensive effort to get at metabolic processes in bone and synovium and possibly even fibrocartilage structures such as the meniscus or labrum may provide valuable insights into OA biology.

The BIPED classification contains other uses of biomarkers besides diagnosis. Three other elements of the classification (see Table 1) might actually provide uses for biomarkers in OA that would be clinically meaningful. The most likely are biomarkers for prognosis $(\mathrm{P})$ and efficacy (E). These, in turn, are related to burden of disease (B) because any treatment that might stabilize or decrease the burden of disease might improve prognosis. Because most systemic biomarkers arise from many structures within the body, only some of which are the focus of patient complaints, it makes sense that the blood or urine level of a biomarker may reflect the total body burden of disease perhaps better than it reflects the presence or absence of disease in a given joint. Addison, et $a l^{8}$, for example, recently demonstrated that serum COMP levels correlated with the total body burden of OA as defined by the number of bone scan sites affected.

Even more likely to be advantageous is the use of biomarkers as a measure of prognosis. OA is a dynamic process with tissue damage and attempted repair. Disease progression may occur when damage exceeds repair. If biomarkers are selected that reflect the process of joint destruction and not static processes that have already occurred (like a radiograph), then a biomarker may predict what will happen to a joint or to the patient's OA in general. Similarly, if treatment slows joint damage then the same measures of dynamic destruction would diminish and provide evidence that the treatment was effective. In fact, in $\mathrm{OA}$, where even magnetic resonance imaging may not sensitively reflect the consequences of effective therapy, systemic biomarkers may provide an excellent opportunity to identify whether a treatment is working in treated patients. The prognosis and efficacy of therapy biomarkers are related because they both examine the longitudinal course of $\mathrm{OA}$ and require biomarkers that reflect the dynamic process of disease.

$\mathrm{OA}$ is not unique in providing an opportunity for prognosis and efficacy biomarkers but not necessarily diagnostic ones. Other conditions in which biomarkers are used mostly to evaluate disease prognosis and the efficacy of treatment include prostate-specific antigen for prostate cancer $^{9}$, cancer antigen- 125 for ovarian cancer ${ }^{10}$, and carcinoembryonic antigen for colon cancer ${ }^{11}$. Like OA, these biomarkers do not work well as diagnostic tests, and the reasons are instructive. In all cases, the tests detect too many false positives, persons who either have the disease but do not need treatment or people who have related benign disorders. Because some OA pathology exists in most middle-aged and older persons and much of this is and will remain asymptomatic, identifying and treating all persons with OA based on a diagnostic marker may not be clinically useful.

While prognosis studies are certainly promising, Personal non-commercial use only. The Journal of Rheumatology Copyright @ 2014 . All rights reserved. 
biomarkers that track the efficacy of treatment constitute the most exciting possibility. One reason this is exciting is that advances in imaging to demonstrate treatment efficacy are quite promising. However, the challenges to validation of a biomarker of efficacy, be it from blood, urine, or imaging, are daunting. First, there needs to be an example of an effective therapy tested in a trial in which biomarkers are also measured. Because it is not clear that there are any current structure-modifying treatments for OA, the characterization of a biomarker whose change might parallel disease improvement or disease stabilization is extremely challenging. It is only when such a treatment emerges that opportunities will arise that permit characterization of efficacy biomarkers. In the meantime studies that focus on biomarkers and their relation to disease burden and prognosis biomarkers have the greatest chance of providing salient evidence supporting their role as efficacy biomarkers.

The focus of this editorial has been on systemic biomarkers of OA. OA is usually localized, affecting one or a group of joints. Therefore, the optimal marker of disease may be one that provides information on the disease in a joint. That suggests that imaging and synovial fluid markers may be more informative than systemic markers. Further, these biomarkers are closer to the disease outcomes of interest, especially if those relate to structural deterioration within a joint. Thus, for diagnostic, and perhaps even for prognosis and effectiveness biomarkers, imaging and synovial fluid markers may hold more promise than systemic biomarkers.

\section{ACKNOWLEDGMENT}

We appreciate the suggestions of Dr. Michael LaValley and the technical assistance of Anne Plunkett.

DAVID T. FELSON, MD, MPH,

Clinical Epidemiology Research and Training Unit at the Boston

University School of Medicine,

Boston, Massachusetts, USA;

Arthritis Research UK Epidemiology Unit,

University of Manchester, Manchester, UK; and

UK National Institute for Health Research Manchester Musculoskeletal

Biomedical Research Unit, Manchester Academic Health Science Centre, Manchester, UK.
Dr. Felson is supported by US National Institutes of Health AR47785. Address correspondence to Dr. Felson, Boston University School of Medicine, A203, 715 Albany St., Boston, Massachusetts 02118, USA. E-mail: dfelson@bu.edu

\section{REFERENCES}

1. Aslam I, Perjar I, Shi X, Renner J, Kraus V, Golightly Y, et al. Associations between biomarkers of joint metabolism, hand osteoarthritis, and hand pain and function: The Johnston County Osteoarthritis Project. J Rheumatol 2014;41:938-44.

2. Kraus VB. Do biochemical markers have a role in osteoarthritis diagnosis and treatment? Best Pract Res Clin Rheumatol 2006;20:69-80.

3. Hoch JM, Mattacola CG, Medina McKeon JM, Howard JS, Lattermann C. Serum cartilage oligomeric matrix protein (sCOMP) is elevated in patients with knee osteoarthritis: a systematic review and meta-analysis. Osteoarthritis Cart 2011;19:1396-404.

4. van Spil WE, DeGroot J, Lems WF, Oostveen JC, Lafeber FP. Serum and urinary biochemical markers for knee and hip-osteoarthritis: a systematic review applying the consensus BIPED criteria. Osteoarthritis Cart 2010;18:605-12.

5. Bauer DC, Hunter DJ, Abramson SB, Attur M, Corr M, Felson D, et al. Classification of osteoarthritis biomarkers: a proposed approach. Osteoarthritis Cart 2006;14:723-7.

6. Cibere J, Zhang H, Thorne A, Wong H, Singer J, Kopec JA, et al. Association of clinical findings with pre-radiographic and radiographic knee osteoarthritis in a population-based study. Arthritis Care Res (Hoboken) 2010;62:1691-8.

7. Criscione LG, Elliott AL, Stabler T, Jordan JM, Pieper CF, Kraus VB. Variation of serum hyaluronan with activity in individuals with knee osteoarthritis. Osteoarthritis Cart 2005;13:837-40.

8. Addison S, Coleman RE, Feng S, McDaniel G, Kraus VB. Whole-body bone scintigraphy provides a measure of the total-body burden of osteoarthritis for the purpose of systemic biomarker validation. Arthritis Rheum 2009;60:3366-73.

9. Prostate-specific antigen (PSA) test. National Cancer Institute 2012. [Internet. Accessed March 6, 2014.] Available from: www.cancer.gov/cancertopics/factsheet/detection/PSA

10. Schmidt C. CA-125: a biomarker put to the test. J Natl Cancer Inst 2011;103:1290-1.

11. Duffy MJ, van DA, Haglund C, Hansson L, Klapdor R, Lamerz R, et al. Clinical utility of biochemical markers in colorectal cancer: European Group on Tumour Markers (EGTM) guidelines. Eur J Cancer 2003;39:718-27.

J Rheumatol 2014;41:834-6; doi:10.3899/jrheum.140094 